\title{
Position: Visual Sentences: Definitions and Applications
}

\author{
Mark A. Livingston and Derek Brock
}

Index Terms—Charts, Diagrams, and Plots; Comprehension, Non-verbal Communication

\section{A Need for Visual Sentences}

Visual forms of communication are ubiquitous in media, educational texts, government reports, and scientific publications. In military contexts, operators and decision makers frequently rely on information that is presented in visual formats, often in the form of a statistical graph. Two challenges immediately come to mind and drive the research agenda we have been following. We need to know that these graphs are provably understandable. We also need to know that the personnel have the skills to understand the information being presented when it is presented well.

Both of these questions continue to attract research attention. There is abundant literature on how to create graphs that communicate clearly (e.g., $[2,5,11,27,28])$. There are several proposed tests to assess an individual's level of skill in reading graphs; offerings include the Visualization Literacy Assessment Test [12] and Graph Literacy Scale [7]. Notably, many of the proposed tests (including these two) build on Bertin's taxonomy of tasks performed with graphs [2] and then use a subjective writing process to create test items. We wanted something objective, but also something that would allow for a more algorithmic and (if possible) automated test development process.

In this paper, we assert that the right way to achieve both goals relies on perceiving what are the visual equivalents of sentences from graphs.

\subsection{Defining When Graphs are Presented Well}

Among the literature referenced above, we think Kosslyn [11] addressed graph understanding from the best methodology and level of detail. To identify design flaws that limit the readers' understanding, he decomposed graphs into components. As he observes, the three required components (Fig. 1): framework (axes, including tick marks and grid lines), specifiers (bars, points, lines, etc.), and labels (including titles) are intrinsically linked:

"In most cases, the framework serves to organize the display into a meaningful whole.... The specifier conveys the particular information about the entities represented by the framework. The specifier serves to map parts of the framework (actually present or inferred by the reader) to other parts of the framework.... The labels are composed of letters, words, numbers, or pictures and provide an interpretation of a line or region (which is a component of either the framework or the specifier)."

Kosslyn goes on to apply three levels of analysis to graphs: syntactical, semantic, and pragmatic. These perspectives are drawn from the study of linguistics (e.g., [9]). According to Kosslyn's analysis, at the syntactic level, a symbol should be distinguishable and arranged properly relative to other symbols. At the semantic level, it should be uniquely interpretable. At the pragmatic level, potential flaws discovered at the syntactic and semantic levels must be assessed with regard to the

- Mark A. Livingston is with the U.S. Naval Research Laboratory. E-mail: mark.livingston@nrl.navy.mil.

- Derek Brock is a volunteer emeritus with the U.S. Naval Research Laboratory.

This work is licensed under a Creative Commons Attribution 4.0 International License.

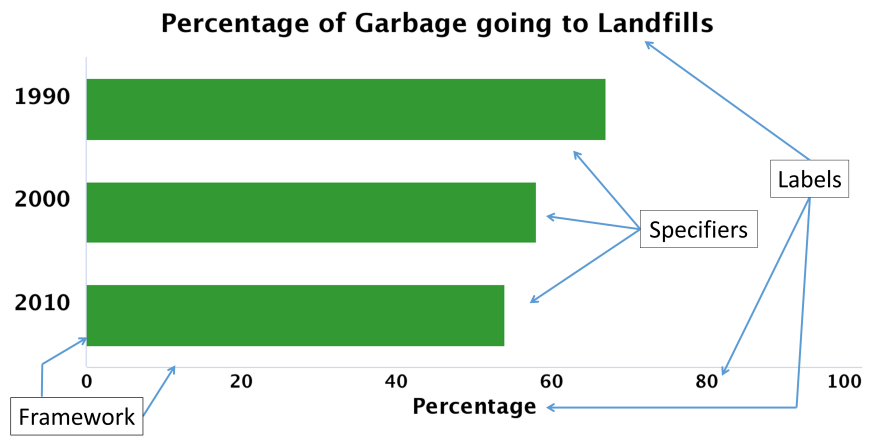

Fig. 1. A simple graph with Kosslyn's components illustrated; we used only specifiers, framework, and labels.

purpose and context of the graph. The purpose and context determine whether the potential flaws create real problems for a reader's ability to understand the graph.

We adopted Kosslyn's decomposition of graphs in order to define what we call visual sentences, but first we motivate our need for them.

\subsection{Increasing Objectivity in Comprehension Assessment}

The issue of objective assessment of comprehension has a long history for prose reading. We knew of one technique for which the objectivity of testing was a central goal. The Sentence Verification Technique (SVT) $[22,24]$ uses one of the basic building blocks of prose (a sentence) to construct test items (queries) to assess the reader's comprehension of a text passage. In particular, it was designed to mitigate the influence of prior knowledge and logical reasoning ability as pathways to correctly respond to queries. These influences were recognized as problems at the time of the SVT development [23] and continue to be recognized $[8,20]$.

The SVT is based on two premises [24]. First is "that language comprehension is a constructive process in which an incoming linguistic message, the recipient's prior knowledge, and the environmental context in which the message is received all interact to determine the nature of the comprehended message." Second is "the memorial representation of this comprehended message is thought to be in a form which preserves the meaning of the message but not its surface structure." The SVT asks not for factual statements or inferential conclusions the reader reaches. Rather, it asks the reader to verify whether a query repeats information given in the source passage or introduces new (including conflicting) information. The authors constructed four types of sentences, collectively designed to defeat skills and knowledge from outside the source text that confound comprehension: rote memory, prior knowledge, and logical reasoning.

The four query types are termed original (verbatim copy of a source sentence), paraphrase (an equivalent rewording of a source sentence), meaning change (a source sentence with a single modification that clearly alters its functional meaning), and distractor (a sentence with new information, consistent with the topic and style of the source material). The first two types give information consistent with the source, whereas the last two types give information not consistent with the source. Note that a meaning change could negate a source sentence, or it could alter the context in a way that could be consistent with the 
source (but is not).

Pearson and Hamm [17] reviewed reading comprehension assessment methodologies, noting that "SVTs can be created using a procedure that involves relatively few inferences and judgments on the part of the test developer." This objectivity in test development is, as noted above, important to our goals.

\section{Identifying Visual Sentences in Graphs}

We wanted our graphs to be demonstrably understandable. We argue that specifier(s), framework, and labels are necessary and sufficient to form complete information statements - visual sentences - from graphs. Kosslyn's components (individually) do not convey complete thoughts. In effect, they function akin to words in a sentence, whereas complete thoughts are coordinated, collectively, by the graph's components. A single data point, divorced from a graph, is not an information statement; but it becomes one when it coordinates values along two axes in a framework, elucidated by the corresponding labels. Two bars from the same graph could express an abstract relationship, but fail to make a meaningful statement until that relationship is expressed in terms of the metric of the framework and labels. Kosslyn demonstrated sufficiency and argued the necessity of these components to convey understanding to the reader. He then identified when this coordination broke down due to missing or misleading uses of the components. We leverage his work to identify the multitude of ways to identify visual sentences embedded in graphs.

We felt that we did not need or want to concern ourselves with graphs that were missing specifiers, framework, or labels. (Although both we and Kosslyn require them, certainly examples of such "deficient" graphs exist.) It is fair to assert that a specifier and labels together could make a complete statement, but only if the labels implicitly indicate the framework. One example of this would see value labels taking the place of a dependent axis, giving the reader a tool (in Kosslyn's terminology) to infer the framework. In another example, labels in graph or axis titles might identify a quantity as a "percentage" and thus enable a reader to infer missing labels for the minimum and maximum. In both of these examples, one could apply the inferences described and achieve comprehension of the desired message. But inevitably, some readers (likely those with less experience reading graphs) would fail to receive the message correctly. One of our goals is testing a general population for the skill of graph comprehension; a graph missing one of the components would require a high level of skill or require prior domain knowledge. A test item from such graphs would likely have low discriminability or be confounded by prior knowledge. This would not further our testing goals.

Now we have shown how we identify visual sentences and determine whether they are complete. This enables evaluation of graph correctness. We focus next on assessment of the reader's comprehension.

\section{Visual Sentences to Assess Graph Comprehension}

We needed an objective construction of test items for assessing graph comprehension. The SVT was designed to mitigate the challenges raised by use of rote memory, prior knowledge, and logical reasoning to respond to reading comprehension test items. We contend that these issues confound graph comprehension assessment; therefore, we adapted the SVT to create test items for graph comprehension. In this effort, we were encouraged by early work that used pictures depicting events in place of sentences in a verification task akin to the SVT $[19,23]$, noting evidence that pictures and verbal material are frequently translated into the same or similar memorial representations. This memorial representation is what one tests with the SVT. We had successfully applied the SVT to listening comprehension [3], and to comprehension of node-link diagrams as part of a multimedia document [14]. Using visual sentences, our task became to determine how each of the graph components could be, should be, or must not be modified in a manner consistent with the goals of the SVT. Thus we devised a set of rules that addressed the changes that are permitted, mandatory, and prohibited for specifiers, framework, and labels [13].

When the SVT is used with prose, one sentence is selected from a few paragraphs of text. The SVT construes a prose sentence as a basic
The percentage of garbage going to landfills in 2010 was $54 \%$.

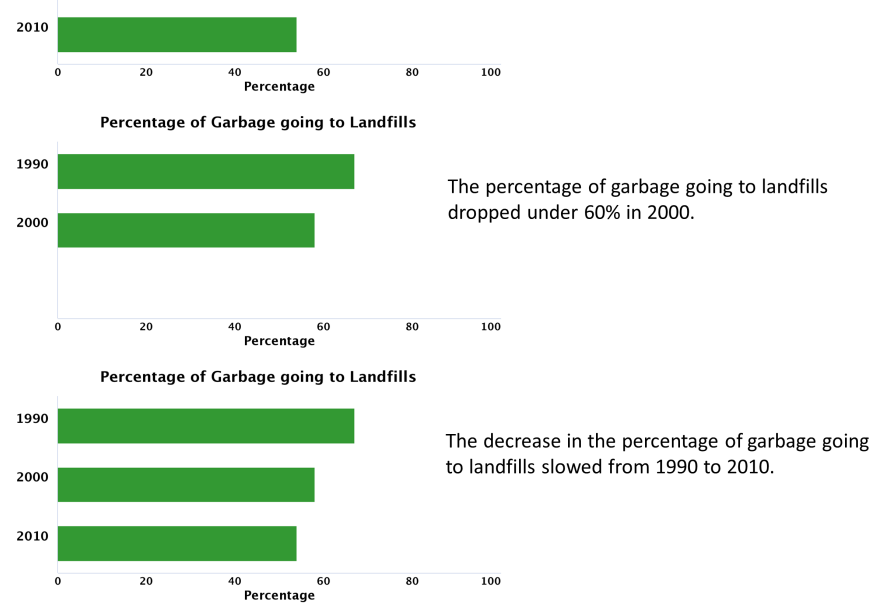

Fig. 2. Left: Example visual sentences from the graph in Fig. 1. Additional visual sentences are present in the original graph. Specifiers and labels that are not relevant to a given visual sentence have been removed. Right: Prose characterizations corresponding to the visual statements on the left (other descriptions are possible). The progression of visual sentences shown here, ranging from simple to complex, illustrate Bertin's taxonomy of graph tasks [2].

unit of communication. (One could debate whether one independent clause from a compound sentence could be the basis of a query.) An analogous question for a graph is 'How many data points should be included in a visual sentence?' We developed rules that define visual sentences as ranging from a single datum to the entirety of a graph. In our initial research, we chose to work with comparatively simple graphs and settled on four rules that limit the complexity of visual sentences for our tests. We can select all points that share a value on the $x$-axis, all points that share a value on an axis implied by a legend (a series, in some common graph terminology systems), or sub-select from one of these two sets (but not from both).

This richness owes to the multiple ways one can build a visual sentence from a graph. A single point gives a statement about one value. Two or more points can make a compound statement about values, or they may make a statement that compares the values (with or without explicit reference to them). A series can make a statement about a trend or a prediction. These statements, respectively, echo the elementary, intermediate, and overall reading levels (Fig. 2) that Bertin [2] posited for graphs, which are often used as a basis for queries on graph comprehension tests developed by subject matter experts [7, 12]. They give the same task range to our queries (Fig. 3).

For a test item that is a meaning change query, we developed rules that specify the minimum for how far points must be moved. Recall that a meaning change modifies a sentence in the source so that when posed in a query, it is not a restatement of information contained in the source. We need these changes to be unambiguous, so we move a selected point a minimum fraction of the graph's dependent axis. We currently use one-third, although this is admittedly experimentally determined as a good rule of thumb. (An earlier version used the distance between gridlines or labels.) Similarly, points introduced for distractor queries must be one-third of the distance away from the points to which they are analogous. For queries that use multiple points, we relax the requirement to apply to a single point that significantly changes the comparison or trend, using the minimum of one-third displacement for at least one point. We considered rounding or smoothing of data, but decided this was unwise.

Another design decision concerned the non-data-carrying appearance parameters of the graph. Should we allow changes to the color 

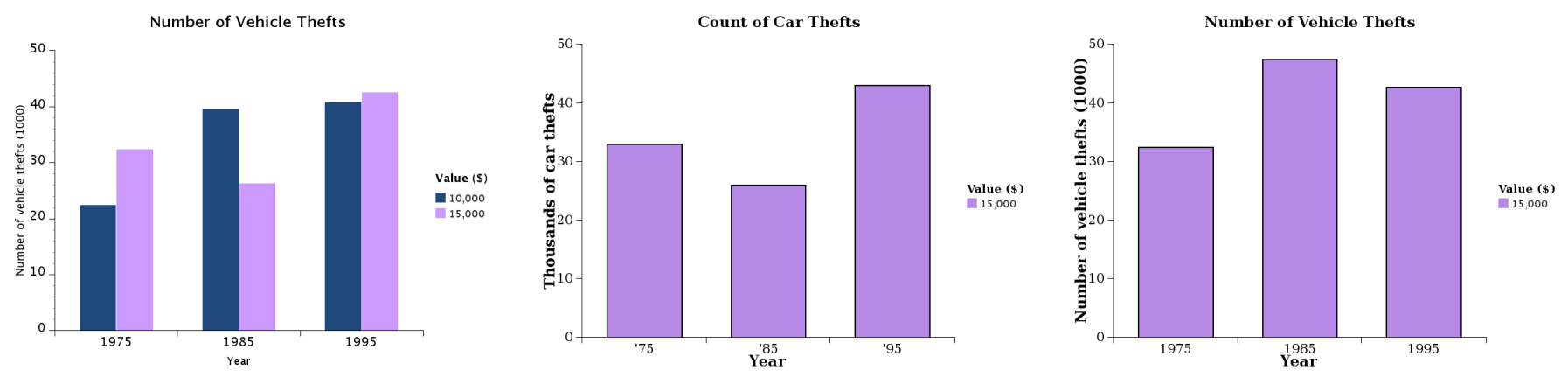

Number of Students Enrolled in Computer Science Classes
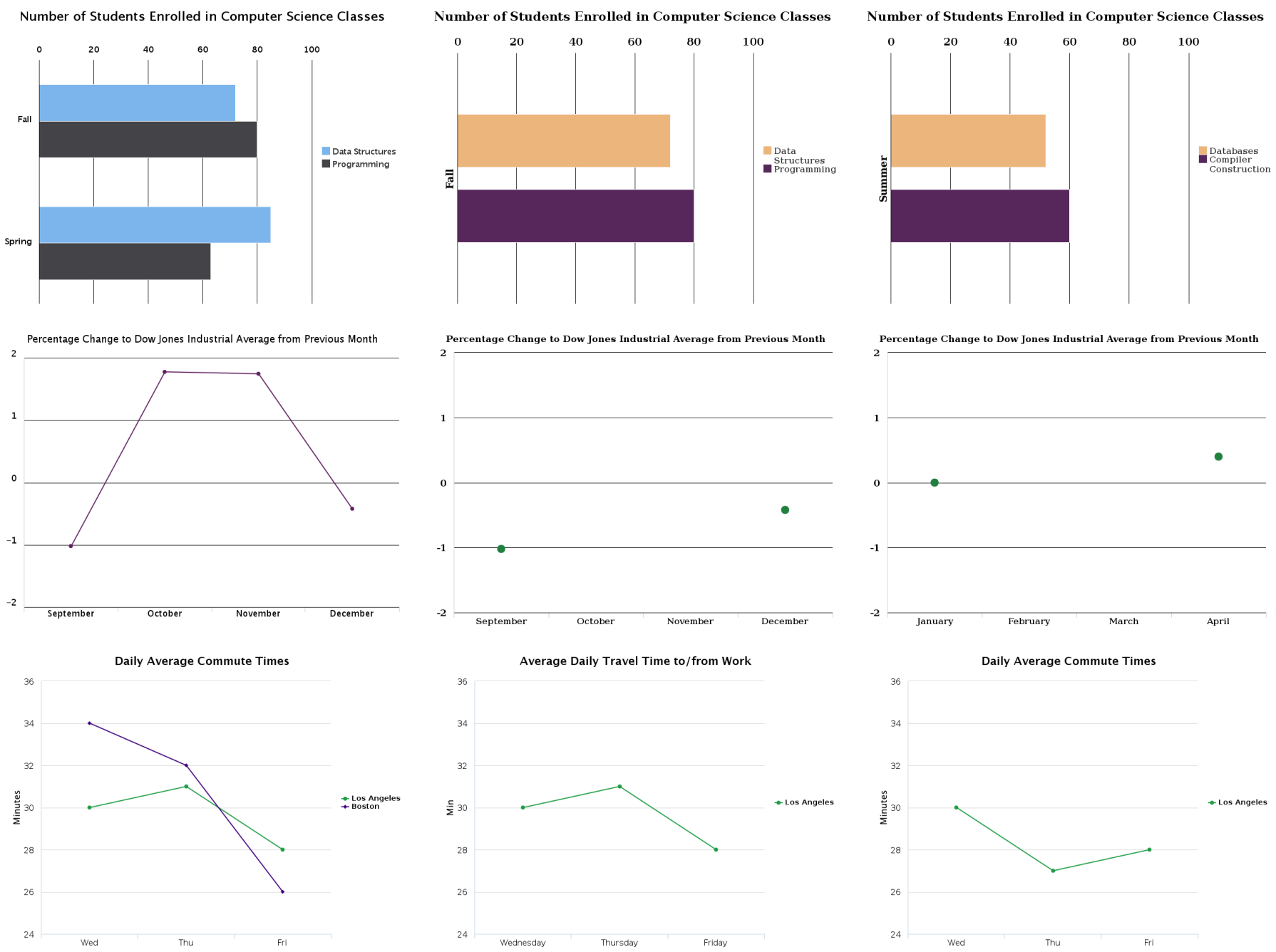

Fig. 3. Visual sentences allow us to generate SVT-like graphical queries in a algorithmic way and evaluate a reader's comprehension of information presented in a graph. Each row shows a source graph at the left and two example queries based on the source in the middle and right columns. Much like a prose sentence can be formatted in many ways without altering its content, these queries are superficially modified. Like the SVT, the response task associated with each visual query is whether the "sentence" it presents agrees with the information in the source graph or not.

Top row: Queries based on the $\$ 15,000$ series in the source. The paraphrase (middle) maintains the original data relationships but uses different words and conventions for the labels with essentially the same meaning as those in the source. In the meaning change (right), one of the specifiers (the value for 1985) is nearly double its value in the source graph.

Second row: Queries based on the data in the Fall category on the vertical axis in the source, with superficial modifications to color, font, etc. The original (center) depicts a verbatim copy of the Fall data in the source. In contrast, the distractor (right), presents a wholly new visual sentence, albeit consistent with the informational theme of the source. This latter query can be recognized as a distractor (i.e., as new information) because neither the named category on the vertical axis, the series names in the legend, nor the values depicted in the body of the graph are present in the source.

Third row: Queries based on two points in the source, with superficial formatting changes (marker size and color). The two values depicted in the original (center) are the same as their counterparts in the source graph. The distractor (right) introduces categories on the horizontal axis and plots values in the graph that are thematically consistent with but not present in the source.

Fourth row: Queries based on the Los Angeles series in the source. The paraphrase (center) maintains the source data relationships but uses different words and conventions for the labels with essentially the same meaning as those in the source. In the meaning change (right), the specifier for Thu shows a smaller value than the corresponding value in the source; this inflects the corresponding data relationships compared to the source. 
map? changes to parameters such as line width, (bar) border width, or (point) marker type? We answered all of these in the affirmative. We reasoned that we (and the original SVT) could in theory change the font (family, size, or weight) or the SVT could change the alignment of the query sentence with respect to the margin. Such changes would not change the meaning from the source passage. So we allowed these changes, even in the "verbatim" original queries. Once applied to the original query, we applied them in exactly the same way to the corresponding paraphrase, meaning change, and distractor queries.

Labels presented a vexing challenge. Ideally, we want to change them in paraphrase queries, so as to change "as many words as possible ... without altering the meaning" (as Royer et al. [24] defined). However, this costs us our goal of automatic query generation. We need manual labor to make such changes, as we do not regard natural language processing to be sufficiently reliable to make such changes automatically. Our automated system can edit labels to or from a few standard abbreviations (e.g. months, days of the week, etc.). Our early manually-written queries do have changes to labels in paraphrase queries, so our objection is purely practical rather than theoretical.

Frameworks present another set of challenges. Would changing the range of an axis be changing the syntax of the graph? We believed that it would not, but this change was only allowed to make a meaning change or a distractor query. Certainly more stark changes, such as changing to or from a logarithmic axis or transposing the graph, would be a change in the graph's syntactical structure and thus are not made for any query. We consider changes to tick marks and grid lines to be acceptable in a paraphrase, since these do not change the central message; however, one could argue this point. The potential to emphasize certain values or more easily compare certain values might change the relative importance of the (multiple) statements made by a graph, if not adding new statements.

To further our goal of automation, we mapped the rules for forming visual sentences and changing the appearance of queries onto a graph specification language. Text is easier to manipulate than an image. Our clients were using the HighCharts ${ }^{1}$ specification, so we adopted it and implemented a rule-based system that edits specifications (with inhibition of conflicting rules). It can also invoke a rule to alter labels, though (as noted above) this is done manually.

\section{Visual Sentences in Other Media}

In earlier work [14], we included a diagram and tables in a multimedia source reading for which we wanted comprehension queries. Kosslyn also discusses the components of a node-link diagram, using the same terminology. The framework consists of the nodes, while the specifiers are the edges that connect the nodes. Labels again help interpret the framework or specifiers. Using this decomposition, we can again derive sentences. Without knowing Kosslyn's decomposition, we independently arrived at the conclusion that our nodes were nouns and our edges were verbs linking actors to actions or beliefs. We thus reasoned that we needed at least two nodes, an edge between them, and the labels associated with them in order to construct a sentence. We expressed these sentences both visually and in prose.

We defined rules to transform components within diagrams into SVT queries (Fig. 4). All queries selected a connected subset of nodes (framework) and edges (specifiers), with their labels. Original queries made no changes. Paraphrase queries changed the labels of nodes to nouns we judged subjectively to be equivalent. Meaning change queries sometimes substituted one new label and sometimes changed one edge type, but not both. Distractor queries changed at least two of the set composed of the labels and edge types.

Our tables held text in cells. Some held complete sentences (including multiple sentences); others had lists of nouns. However, when combined with the respective row and column header, we found it natural to write a sentence that expressed a statement equivalent to one contained in any cell. Thus we again could write queries in the style of the SVT. We did not include multiple cells in one query or require that the entire contents of a cell be used in a query. It is arguable whether

${ }^{1}$ https://api.highcharts.com/highcharts/ all text in a cell should be regarded as part of a single sentence. We asserted that it did not, just as a single point in a data series connected by a line may make a statement on its own.

Because the sentences associated with a table are somewhat more rigidly constrained by the table structure, our rules for query formation (Fig. 5) focused on changing (or not changing) the labels within a cell (not row or column headers). We did not alter any structural element of the table; we did not have any empty cells in the table to fill in for a query, nor did we form a query by emptying a cell. These would be changes to the specifiers. We did not change the framework by adding or deleting rows or columns, nor did we change the header-subheader structure for rows or columns.

Our volunteer readers were better at answering queries about the diagram than they were about the accompanying prose or accompanying tables. Full details of our results are available elsewhere [15]. We note some evidence that the visual sentences from the diagram and tables were being processed like verbal sentences. The order of mean response times for queries about prose, about the diagram, and about the tables correponded to the number of information statements we counted in each of our multimedia sources. While this was only a single experiment with 32 subjects, we note that propositional density is cited as a factor in the difficulty of reading comprehension tests [10]; this ordering of response time is consistent with that observation.

\section{Applications of Visual Sentences}

For our goal of using the methodology of the SVT to assess graph comprehension, the implication of being able to form sentences from graphs (or diagrams or tables) is clear. It is critical to the ability to objectively and efficiently develop a large number of test items. Because of the structure of the SVT and the rules we devised to adapt to the visual sentences, we believe that our queries are more objective. Obviously, there is subjective test design created by which questions are selected, though we believe this can be mitigated by uniform distribution in application of the rules. We need to be able to automatically or at least algorithmically generate sufficient queries in order to be able to assess learning. Measuring improvement requires new material that is (to within our ability to make and measure it) of equivalent difficulty. While the accepted process of test development works well in the long-term, it requires significant effort and has a relatively low yield of questions that pass the psychometric criteria. Even then, questions can make it through that process and still be found to be of questionable validity for a variety of reasons. While our test development methodology cannot guarantee that all questions will achieve validity, the rules we developed enable a largely automated implementation and mitigate some of the major pitfalls. This will assist with generating a greater number of questions; we further believe that using the rule-based system, we can learn which rules (or combinations thereof) create valid questions at desired levels of difficulty, although this remains to be proven through extensive testing with queries generated through our SVT-based methodology.

We have seen that the visual sentences that are present in a graph readily reflect Bertin's taxonomy of elementary, intermediate, and overall reading levels [2]. A visual sentence can focus on an individual data point (elementary reading), compare data points (an intermediate reading), or describe trendsor anomalies (an overall reading). Thus there is a correspondence between the queries of an SVT using our visual sentences and queries on previously published graph comprehension tests.

The SVT explicitly tests the representation of the (verbal or visual) statements in the memory of the reader. The graph comprehension tests we have seen in the literature leave the source material present for reference. This difference results from different opinions on the definition of comprehension [10,23]. While we leave that debate to the cognitive science community, the SVT methodology mitigates the effects of both referencing the source passage without achieving comprehension and answering by using rote memory without achieving comprehension. We believe this contributes to the objectivity of test questions developed in the SVT methodology; this objectivity was a motivation for our visual sentence definition. We observed a correlation 

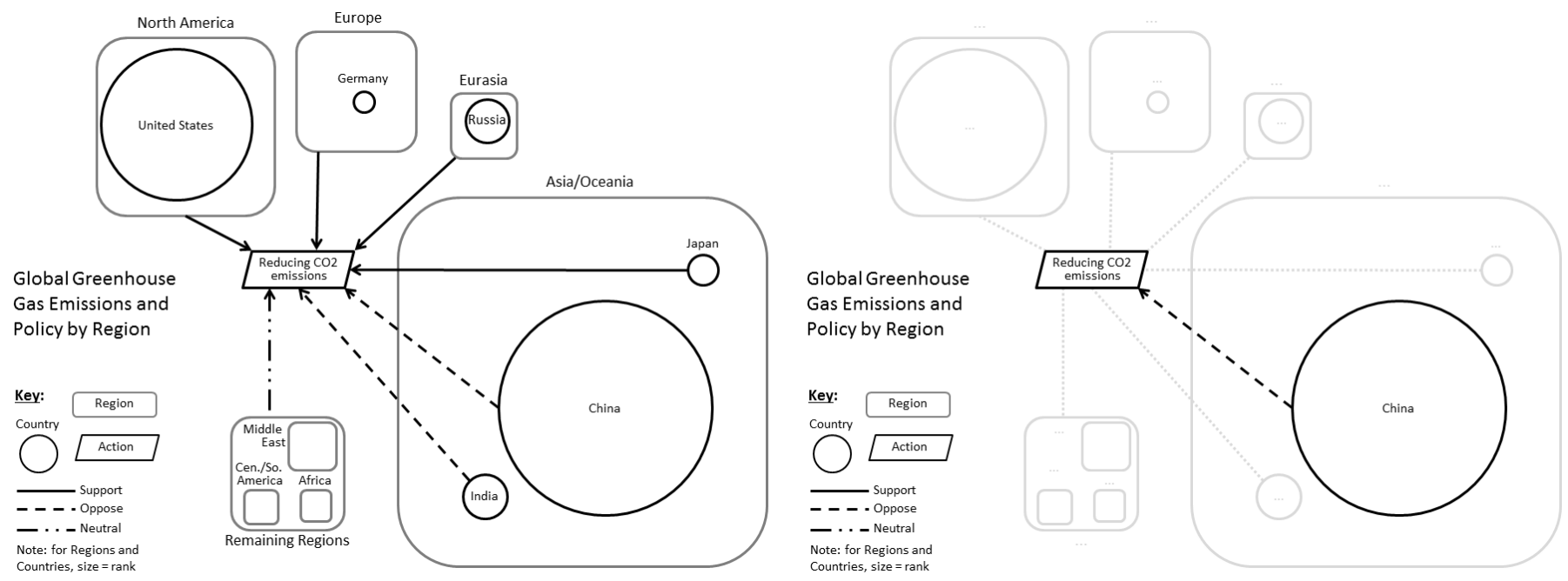

Fig. 4. Left: A source diagram from which we extracted sentences to query comprehension; this diagram accompanied a prose narrative that included two tables (cf. Fig. 5). Right: A visual sentence extracted from the diagram consisted of at least two nodes and the edge between them. This connection between two entities or activities forms a complete thought. The particular sentence shown here forms an original query per the rules of the SVT. No changes were made to the nodes and edge that form the sentence, although the other nodes and edges were made less salient, and other labels were removed. For a paraphrase query, rules might dictate to change the entity labels. For a meaning change query, rules might dictate to change a label or change the edge type to something else already specified in the legend. For a distractor query, rules would dictate a change to introduce new labels. We do not alter the legend to alter the sentence. This would be akin to redefining the language used in the sentence as a way to query comprehension, so we avoided it.

\begin{tabular}{|c|c|c|c|c|}
\hline & Significant types & Supply & Environmental issues & Policy notes \\
\hline Fossil & $\begin{array}{l}\text { - Petroleum } \\
\text { - Coal } \\
\text { - Natural Gas }\end{array}$ & - Growing reserves & $\begin{array}{l}\text { - Extraction risks and by- } \\
\text { products } \\
\text { - Main source of } \\
\text { greenhouse gases and } \\
\text { air pollution }\end{array}$ & $\begin{array}{l}\text { - Dominant worldwide } \\
\text { energy sector }\end{array}$ \\
\hline Renewable & $\begin{array}{l}\text { - Hydro } \\
\text { - Solar } \\
\text { - Wind } \\
\text { - Biofuels } \\
\text { - Geothermal }\end{array}$ & - Theoretically unlimited & - Few risks overall & $\begin{array}{l}\text { - Smallest worldwide } \\
\text { energy sector }\end{array}$ \\
\hline
\end{tabular}

\begin{tabular}{|c|c|c|c|c|}
\hline & $\cdots$ & $\cdots$ & $\cdots$ & Policy notes \\
\hline Fossil & $\cdots$ & $\cdots$ & $\cdots$ & $\begin{array}{l}\text { - Dominant worldwide } \\
\text { energy sector }\end{array}$ \\
\hline$\cdots$ & $\cdots$ & $\cdots$ & $\cdots$ & $\cdots$ \\
\hline$\cdots$ & $\cdots$ & $\cdots$ & $\cdots$ & $\cdots$ \\
\hline
\end{tabular}

Fig. 5. Top: A source table from which we extracted sentences to query comprehension. This table was part of the narrative mentioned in Fig. 4. Bottom: A visual sentence extracted from the table used contents from a single cell to connect the concepts embodied in the row and column header. This forms a complete thought. In this particular sentence, there was one phrase in the cell; other sentences could be formed by using multiple items from a list in a cell (e.g., the top row of the first column) or by using a single list element from a list (e.g., middle row of the third column). This sentence forms an original query per the rules of the SVT. No changes were made to the cell contents. A paraphrase query would change the cell contents to other words with equivalent meaning. A meaning change query would change the contents so as to mean something different. A distractor query would introduce new concepts or entities into the sentence. As with the legend in a diagram, we chose not to alter row or column headers, although this restriction may be tighter than necessary to form valid queries. It certainly restricts us from valid variations of sentences. Also, we similarly made the cells not included in the sentence less salient, but did not completely remove them. 
between the study time our graph readers spent on graphs and the number of visual sentences we counted in each, suggesting that visual sentences may be processed similarly to prose sentences, for which the number of propositions determines the time required to read [10].

There are applications of this idea that we think have merit for other research involving graphs. Acartürk [1] asked volunteers to provide verbal descriptions of graphs with and without graphical cues, which emphasized certain elements. Our method of generating visual sentences from graphs might help develop a more objective rubric for such a protocol. In another research approach, there is a significant amount of work in automatically summarizing graphs or documents containing them. Reasoning about graphs and forming hypotheses about their meaning can inform readers for whom access to the information may be (temporarily or permanently) challenging, such as users of digital libraries, the visually impaired, or users on devices where graphics are impractical or inaccessible. It may help with search engine retrieval of documents or graphs, improve automated answering of questions about graphs, and connect graphs to related text in multimedia documents. There are many examples of this type of work in a growing field $[4,6,16,21,26]$.

Multiple researchers [18, 25] have noted that the graph type (bar versus line) can influence the interpretation of a graph. We speculate that a system that produces sentences from a graph could ultimately help reveal the primary interpretations that readers are likely to deduce from a graph. Sentences could be ranked in terms of the number of graph components that contribute to them. If particular components require a separate explanation from the main trend of a graph, then that might indicate an important sentence in the graph's message.

\section{Conclusion}

We have posited a formal way to identify visual sentences in graphs. We have used this to build machinery to algorithmically and even automatically generate comprehension queries for statistical graphs. We have shown how the queries we generate can conform to the popular question types derived from Bertin's reading levels. We directly used Kosslyn's components for graphs, and we see that we were following his formula when constructing sentences from node-link diagrams and when constructing sentences from tables. We see other research that could benefit from our insights.

The ubiquity of graphs in society demonstrates that they are considered an effective and efficient method of communication. Our analysis of what constitutes visual sentences, in conjunction with Kosslyn's decomposition, shows why graphs are so powerful. We welcome comments, extensions, and challenges to our ideas.

\section{ACKNOWLEDGMENTS}

The authors wish to thank Mike Royer, Jonathan Decker, Dennis Perzanowski, Joseph Mathews, Christopher Van Dolson, Tucker Maney, Wende Frost, and Alex Lulushi. This work was supported in part by the Naval Research Laboratory Base Program.

\section{RefERENCES}

[1] C. Acartürk. Towards a systematic understanding of graphical cues in communication through statistical graphs. Journal of Visual Languages \& Computing, 25(2):76-88, Apr. 2014.

[2] J. Bertin with M. Barbut et al. Sémiologie Graphique: Les diagrammes, les réseaux, les cartes; revised edition. Gauthier-Villars, 1973. Translated as "Semiology of Graphics" by W.J. Berg, Univ. of Wisconsin Press, 1983.

[3] D. Brock, B. McClimens, J. G. Trafton, M. McCurry, and D. Perznowski. Evaluating listeners' attention to and comprehension of spatialized concurrent and serial talkers at normal and a synthetically faster rate of speech. In Proc. of the $14^{\text {th }}$ Intl. Conf. on Auditory Display, June 2008.

[4] Z. Chen, M. Cafarella, and E. Adar. DiagramFlyer: A search engine for data-driven diagrams. In WWW'15 Companion: Proceedings of the $24^{\text {th }}$ International Conference on World Wide Web, pp. 183-186, May 2015.

[5] W. S. Cleveland. The Elements of Graphing Data. Hobart Press, 1994.

[6] S. Elzer, S. Carberry, and I. Zukerman. The automated understanding of simple bar charts. Artificial Intelligence, 175(2):526-555, Feb. 2011
[7] M. Galesic and R. Garcia-Retamero. Graph literacy: A cross-cultural comparison. Medical Decision Making, 31(3):444-457, May/June 2011.

[8] P. Kendeou, K. L. McMaster, and T. J. Christ. Reading comprehension: Core components and processes. Policy Insights from the Behavioral and Brain Sciences, 3(1):62-69, Mar. 2016.

[9] W. Kintsch and P. Mangalath. The construction of meaning. Topics in Cognitive Science, 3(2):346-370, Apr. 2011.

[10] W. Kintsch and T. A. van Dijk. Toward a model of text comprehension and production. Psychological Review, 85(5):363-394, Sept. 1978.

[11] S. M. Kosslyn. Understanding charts and graphs. Applied Cognitive Psychology, 3(3):185-225, July/September 1989.

[12] S. Lee, S. Hee Kim, and B. C. Kwon. VLAT: Development of a visualization literacy assessment test. IEEE Transactions on Visualization and Computer Graphics, 23(1):551-560, Jan. 2017.

[13] M. A. Livingston, D. Brock, J. W. Decker, D. J. Perzanowski, C. V. Dolson, J. Mathews, and A. S. Lulushi. A query generation technique for measuring comprehension of statistical graphics. In Advances in Human Factors in Training, Education, and Learning Sciences, Proceedings of Applied Human Factors and Ergnomics, July 2019.

[14] M. A. Livingston, D. Brock, T. Maney, and D. Perzanowski. Extending the sentence verification technique to tables and node-link diagrams. In Advances in Human Factors in Training, Education, and Learning Sciences, Proceedings of Applied Human Factors and Ergnomics, July 2018.

[15] M. A. Livingston, D. Brock, T. Maney, and D. Perzanowski. Report on an extension of the sentence verification technique to multimedia documents with tables and node-link diagrams. Technical Report Report IMDA11, Naval Research Laboratory Information Management and Decision Architectures, 2018.

[16] R. R. Nair, N. Sankaran, I. Nwogu, and V. Govindaraju. Automated analysis of line plots in documents. In International Conference on Document Analysis and Recognition (ICDAR), Aug. 2015.

[17] P. D. Pearson and D. N. Hamm. The assessment of reading comprehension: A review of practices_-past, present, and future. In Children's Reading: Comprehension and Assessment, chap. 2, pp. 13-69. Lawrence Erlbaum Associates, Inc., 2005.

[18] D. Peebles and N. Ali. Expert interpretation of bar and line graphs: the role of graphicacy in reducing the effect of graph format. Frontiers in Psychology, 6(1673), Oct. 2015.

[19] K. Pezdek. Cross-modality semantic integration of sentence and picture memory. Journal of Experimental Psychology: Human Learning and Memory, 3(5):515-524, 1977.

[20] S. J. Priebe, J. M. Keenan, and A. C. Miller. How prior knowledge affects word identification and comprehension. Reading and Writing, 25:131-149, 2012.

[21] X. Qian, E. Koh, F. Du, S. Kim, and J. Chan. A formative study on designing accurate and natural figure captioning systems. In Extended Abstracts of the 2020 CHI Conference on Human Factors in Computing Systems, Apr. 2020.

[22] J. M. Royer. Developing reading and listening comprehension tests based on the sentence verification technique (SVT). Journal of Adolescent \& Adult Literacy, 45(1):30-41, Sept. 2001.

[23] J. M. Royer and D. J. Cunningham. On the theory and measurement of reading comprehension. Technical Report 91, University of Illinois at Urbana-Champaign, June 1978.

[24] J. M. Royer, C. N. Hastings, and C. Hook. A sentence verification technique for measuring reading comprehension. Journal of Reading Behavior, 11(4):355-363, Dec. 1979.

[25] P. Shah and E. G. Freedman. Bar and line graph comprehension: An interaction of top-down and bottom-up processes. Topics in Cognitive Science, 3(3):560-578, July 2011.

[26] M. Sharma, S. Gupta, A. Chowdhury, and L. Vig. ChartNet: Visual reasoning over statistical charts using MAC-Networks. In International Joint Conference on Neural Networks, July 2019.

[27] E. R. Tufte. The Visual Display of Quantitative Information. Graphics Press, 1983.

[28] H. Wainer. How to display data badly. The American Statistician, 38(2):137-147, May 1984. 